\section{CONTRIBUTIONS TO CLINICAL SURGERY.}

By Ortrer Pemberton, Esq., Surgeon to the General Hospital, and Lecturer on Surgical Pathology at Sydenham College, Birmingham.

\section{VI.-EXCISION OF THE KNEE.} [Continued from page 961 .]

I PROCEED, therefore, to the narration of further cases, exhibiting, some of them, the ordinary, and others, the least frequent complications, attending this operation.

CASE II. G. W., aged 7, a pale, fair-haired, delicate-looking child, was admitted into the General Hospital, Birmingham, on June 8th, 1857, under my care, with disease of the left knee-joint.

History. The knee has been diseased for the last two years; and on two occasions during that period he has been a hospital patient. The disorder commenced after a slight injury occasioned by a fall. His parents are living, and his brothers and sisters are in good health. He has not had glandular enlargements, nor have the lungs shown symptoms of disease.

Present State. The left knee is enlarged and rounded. There is an elastic elevation, corresponding to the synovial spaces by the sides of the patella. The inner condyle is much larger than natural, as is also the head of the tibia. The leg is semiflexed, and admits of very slight motion, which produces acute pain. The integuments are not ulcerated, and are free from pain, with the exception of the spaces by the sides of the patella above referred to. The muscles of the limb are much wasted, but the bones correspond in length to those of the opposite limb.

The treatment, continued for several months, consisted in the careful adaptation of gutta percha splints to the joint, whilst a splint was also applied to the whole length of the limb, and was furnished.with a foot-piece and screw, so as gradually to straighten the extremity; at the same time, codliver oil and a nutritious diet were prescribed, with a view to the improvement of his general health. At this time the child suffered but little pain, though it was evident, no less from the increased size of the knee than from the wasting of the patient's frame, that the disease was making a gradual progress.

In September, there appeared a swelling above the patella, apparently outside the joint, and situated amongst the tendons of the quadriceps. There was an obscure sense of fluctuation about this, but it was entirely free from pain.

Soon after this time, I was desirous to excise this patient's knee, as I was satisfied of the presence of very extensive disorganisation of the joint, and was of opinion that further delay would not prove advantageous. In consultation, however, with my colleagues, the symptoms were not deemed sufficiently urgent to render immediate operative interference expedient; and consequently, the treatment by rest and general means was continued.

Finding that no improvement had manifested itself, either in the part affected or in the constitution, I resected the joint on December 16th.

Operation. The patient being placed under the influence of chloroform, the usual semicircular incision was carried from one condyle, across the ligamentum patellæ, to a corresponding point on the opposite side. The termination of the incision opened the abscess in the quadriceps, and gave exit to a large collection of pus; the soft parts were then cleared, and the ligamentous tissues divided. Before I was aware of it, the knee had been forcibly bent, and the remains of the crucial ligaments were torn through. This proceeding compelled me to saw off and remove the articular ends of the femur and tibia separately instead of in their connected state. The bones were sawn through with an ordinary amputation-saw, and no spatula was introduced. The patella was not removed, No vessel required a ligature at the time of the operation.

Two hours subsequently, the limb was accurately adjusted, and a modification of the Macintyre splint applied, which admitted of the wound being dressed without disturbance, and the boy being placed on a water-pillow, the extremity was lightly suspended in a swing-cradle. A small vessel proving troublesome during the dressing, it was tied, and the edges of the wound were closed by sutures.

Examination of the Resected Bones. About three quarters of an inch of the femur, and rather less than half an inch of the tibia, were removed. The synovial membrane was everywhere in a condition of scrofulous degeneration. The cartilages covering the prominent surfaces of the condyles were almost entirely destroyed, large pieces being separated or only loosely connected with the bone beneath. The head of the tibia on the inner side was in the same condition in regard to its coverings. The bones themselves were filled with scrofulous matter.

December 17th. He passed a restless night. This morning the pulse was 150 .

Dec. 18th. He had a better night; but was restless since morning; and had disturbed the splint. There was a free discharge from the wound.

Dec. 19th. I was compelled to readjust the splint. Tho sutures were removed. The wound was looking healthy.

Dec. 20th. The child was low and feverish. The pulse was rapid; the countenance pinched; the position of limb was good. I could not learn that he had had a rigor.

Dec. 21st. Prostration was not so great. $\mathrm{He}$ had pain in the left elbow-joint, and was sick.

Dec. 22nd. Suppuration was copious from the wound. He had pain in the right knee as well as in the elbow.

Dec. 25th. He had a rigor whilst being dressed. The discharge from the wound was copious. From this date, he gradually sank, and died on January 4th, 1858 , nineteen days after the operation.

Dissection twenty-four hours after death. The brain was healthy. The lungs were remarkably small in size. The apex of the left lung was studded with a few tubercular deposits in an unsoftened state. Small masses of purulent deposit were scattered throughout both lungs; their size varied from a millet-seed to that of a pea or bean. The heart was healthy. In the pericardium was a large quantity of clear serum. The liver was natural in size: its surface was sprinkled by numerous purulent deposits, of the size of split-peas ; and deeply seated in the upper part of the right lobe was a deposit of the size of a bazel-nut, of a similar character. The remainder of the abdominal viscera were natural.

In the resected joint, the union of the flap in front was firm and satisfactory. The ends of the bones were in perfect appo. sition. On separating their surfaces, they were seen to be covered with imperfectly organised lymph. The textures adjacent were not infiltrated or in any way altered from the natural condition. The femoral vein was next traced carefully upwards from below the popliteal; it was healthy, until within a short distance from Poupart's ligament; at this point was located a plug of lymph, of two inches in extent. The plug imperfectly adbered to the coats of the vein, and was broken up in parts by bloody infiltration. The femoral and popliteal veins of the opposite limb were natural; so also were the iliacs and vena cava inferior.

The cavity of the left elbow.joint was filled up with a thin, dirty coloured pus. The left shoulder was healthy. The right shoulder had a quantity of pus within its cavity, whilst the right elbow was healthy. The right bip was filled with pus; the left was healthy. In the several joints occupied by pus, the cartilages and synovial membranes were unaffected.

CASE III. G. A., aged 8, a delicate strumous looking boy, presented himself to my notice in March 1858, suffering from a long standing disease of the left knee.

History. Six years since, after a fall, the joint first began to give pain, and after much suffering in the earlier stages of his complaint, the malady subsided to its present condition.

Present State. His knee is now semiflexed, partially move. able. The tibia is thrown backwards, and the patella rests firmly on the outer condyle. There is general enlargement of the articular extremities, and the entire aspect of the joint presents a globular appearance. He suffers no pain on movement or on pressure; but the limb is useless, is wasted, and he walks on crutches, and has done so for years.

It was evident in this case that all acute disorder had sub. sided; consequently, any treatment contemplated was with the view of, if possible, remedying the inutility of the limb.

At the urgent request of the boy's friends, and after consultation with my colleagues, I determined to excise the joint, in the hope of obtaining a limb more useful than the one just described. Accordingly, on March 24th, under chloroform, I operated in the ordinary way. Thin slices of the femur and tibia were removed, altogether, rather less two inches, and the patella was likewise taken away. There was smart hæmorrhage, and two small vessels required ligature. The child seemed more than usually depressed after the operation, before 
removal from the theatre, but I attributed this more especially to the chloroform.

In the afternoon following, the limb was carefully adjusted in the ordinary apparatus. Sickness had, however, set in soon after his removal to the ward, and continued more or less severely until the next day. Then it somewhat abated under the influence of remedies, but towards evening it was renewed with increased severity. After this there was no amendment, but the symptoms of depression increased, so that, despite all that was attempted for his benefit, he sarik on the 27 th, three days after the operation.

No post mortem examination was permitted.

Examination of the Sawn Articular Extremities. The synovial membrane was everywhere pulpy and degenerated, but the cartilages were only thinned, appearing in other respects healthy. Where the patella rested on the outer condyle, the cartilage had almost entirely disappeared. The tibia was com. pletely thrown backwards behind the internal condyle.

CASE IV. E. H., aged 30, a housemaid, of strumous appearance, was admitted under my care in the General Hospital, Mlay 7 th, 1858.

History. Eighteen months since she first experienced pain in the right knee, near the inner margin of the patella. 'The joint became weak, and the pain increased slowly in intensity, accompanied by some enlargement of the part. Finding that the disease was advancing, and that she was no longer able to pursue her employment, she sought admission into the Hospital.

Present State. The joint is greatly enlarged, all the bony prominences having disappeared. The integuments are discoloured, and there is an obscure sense of fluctuation conveyed to the fingers on manipulation. There is pain on firm pressure, but this is principally confined to the region over the head of the tibia. With all this, the limb is in a good position, and in one very favourable for anchylosis.

With this view, during the next four months, everything was done in the hope of arresting the progress of the disease Once or twice collections of pus were evacuated in the muscles above and below the knee, and her general health continued satisfactory for some time. It was at length, however, evident that something more must be done; and, accordingly, on October 6th, five months after her admission, at her own request, $I$ excised the joint in preference to amputation. The operation was accomplished in the ordinary way. The first incision gave exit to a large collection of pus, situated in the muscles around the joint, and there was also a large quantity in the joint itself, and in the popliteal space. The amount of bone removed measured about one inch and a quarter. The patella was likewise taken away. The hæmorrhage was inconsiderable, and but one vessel required a ligature. There was some sickness soon after the operation, but by the time the limb was adjusted, the patient had very fairly rallied from its effects.

Examination of the Sawn Extremities. The slice removed from the femur is three parts of an inch in thickness. The inner condyle has its articulating surface entirely denuded of cartilage, a slight gelatinous coating existing in one or two spots. The outer condyle is also denuded, with the exception of about a quarter of an inch posteriorly, where the cartilage is much attenuated. The slice from the tibia measured rather less than half an inch. The semilunar cartilages are totally destroyed, with the exception of a small portion behind the spine. The cut surfaces presented the usual appearances of spongy bone. In the slice from the tibia, the saw just passed below a softened portion. The compact layer of bone beneath the cartilages was almost entirely destroyed, and a good deal of new bone had been thrown out around the articular extremities. The synovial membrane was utterly gone. The crucial ligaments were entire, but imbedded in a pultaceous purulent mass. The patella had its cartilage destroyed, and the bone beneath was in a softened state.

For some days after the operation the patient did well. The wound suppurated, and her strength returned.

October 1lth. Had a shivering fit; and two days afterwards a severe attack of erysipelas around the wound and up the thigh manifested itself.

Having recovered from this, on the 30 th, without any preliminary fever, she broke out with confluent small-pox, notwithstanding the knee looked well, and suppurated healthily. There was great depression following the subsidence of the eruption; and the wound discharged most profusely. At the beginning of December she had night-sweats and cough, with purulent expectoration; and on the $22 \mathrm{nd}$ of this month she died, under the wasting influences of diseased lungs.

In the examination of the excised joint, there was found to be not the least attempt at bony union. Pus had burrowed its way in all directions, and had separated the periosteum for some little distance beyond the sawn extremities. These at their opposed surfaces were covered by a sloughy purulent membrane. There were no evidences of purulent absorption. The apices of both lungs contained large cavities, and there was abundant tubercular deposit scattered throughout the other parts of these organs.

CaSE v. C. C., aged 17, a dark-eyed, sallow complexioned Irish girl, was admitted under my care in the General Hos. pital, on July 30th, 1858.

History. About four years since, in a fall, she injured the left knee, and suffered for some time afterwards from pain and lameness. She went about, however, and did not experience great inconvenience until about three months before her ad. mission; then she had constant pain, the joint became swollen, and she was unable to use it in the least degree.

Present State. The joint is greatly enlarged, rounded, and even with the surface, shiny and reddened. The limb reposes on its outer side, and is semiflexed. The least movement occasions intense agony, and there is evident fluctuation in the articular cavity. The patient's general health, owing to long continued pain, is very much reduced. She entreats that something may be done for her relief, but refuses, both by herself, and by her friends, to submit to amputation. After waiting, in the hope that her general health might improve, and finding that hectic was rapidly setting in, on the 19th of August I excised the knee under chloroform. On opening the joint, a pint of pus poured out, whilst there was a second collection in the bursa above the patella. Very thin sections, half an inch of the femur, and the same amount of the tibia, were removed. The patella was left. The superior internal articular artery alone required ligature. For some hours afterwards the prostration was very great, but towards evening reaction was fully established.

Examination of the Sawn Articular Extremities. The synovial membrane was everywhere thickened, and converted into a reddish fungus-like material. The cartilages on the femur, on the tibia, and the semilunar cartilages, were almost entirely destroyed, but the bone was scarcely penetrated by disease in any part. The crucial ligaments were utterly disorganized.

There was no drawback to the steady improvement of the health of this patient, after the operation, for the first two months. The wound progressed favourably, though the suppuration, as might be expected, was of an extreme character.

In November, some abscesses formed in the cellular membrane of the thigh and leg, and required free incisions for their relief; in their presence, however, the wound closed in.

At the end of three months the splints were removed, and the limbs simply supported by a roller. There was now great consolidation about the articular extremities, but no bony union.

At Christmas, four months after the operation, she was permitted to get up and about on crutches. There was unwieldy enlargement about the joint, and decided thickening of the femur.

In February 1859, the enlargement of the thigh had become more decided, and it was accompanied by a good deal of pain. After a time, some abscesses broke of their own accord, and sinuses resulted. Through these a probe could be passed on to the enlarged bone, but I was unable to detect any necrosed portions. She can now walk about fairly with the aid of a leather case and her crutch.

From this time, to a period occupied by the next three months, her progress varied but little. Sometimes she would suffer from the formation of fresh gatherings about the thigh, and require rest and attention, but the general character and power of the limb improved in all respects.

On June 15th, ten months from the operation, the following note was recorded. There is no shortening whatever of the limb, she can walk with facility, and requires only the aid of a stick or single crutch. The soft parts are somewhat swollen and thickened over the upper part of the tibia, and along the inner margin of the seat of incision, where are situated the apertures of one or two sinuses. The thigh bone, as high as the trochanter, is greatly enlarged, and there are the apertures of three or four sinuses, but no necrosed bone can be detected. There is slight movement allowed between the sawn extremities. A week after this she left the Hospital. 
CASE vI. A. H., a labourer, aged 27, was admitted under my care on May 1st, 1859, suffering from long standing scrofulous disease of the right knee. He was pale and thin, being wasted from much pain ; but otherwise he was in good health.

History. Eight years back, the disease originated in a blow. He was under surgical treatment for various periods during this time; and, about two years since, was under my care in the hos pital. At that time, the use of issues, and absolute rest to the limb, sufficed to control the disease; and he was enabled to leave at the end of two months, free from pain; and to get about by the aid of crutches and a support for the knee. He remained well, with the good prospect of a stiff knee, for eighteen months. It then again became painful, and increased in size, so that, his health beginning to suffer, he determined to sulmit to amputation, and accordingly sought the hospital once again with this view.

On examination, the joint was observed to be evenly en. larged, all the bony prominences having disappeared, and an uniform whitish pulpy appearance being presented. The limb was straight; but the least movement of the joint, or pressure of the patella, occasioned acute pain.

May 18th. I excised the joint, in preference to amputation. The operation was accomplished in the customary manner, half an inch of the femur, and the same amount of the tibia, being sawn off, and the patella being likewise removed. There was rather abundant hæmorrhage, a large superior external articular artery requiring a ligature.

Examination of the Resected Parts. The excised parts pre. sented an appearance of profound destruction of cartilage. The faces of the condyles, and the corresponding surfaces of the tibia, were hollowed out by the extension of mischief, and the bony structure adjacent was rendered of a spongy character. The under surface of the patella was similarly affected. There was no trace of the synovial membrane remaining. There was no collection of pus within the joint, but there was a large gathering of matter freed in the course of the incision by the side of the tibia.

For the first few days following he suffered from severe constitutional disturbance, and from a threatening of erysipelas. These drawbacks having subsided, he was doing well, when, on the 24th-six days after the operation-he was seized with alarming arterial hæmorrhage from the situation of the ligature on the superior external articular vessel, which had been thrown off. Before assistance could be rendered, he had lost nearly two pints of blood, and, but for a timely fainting, would in all probability have died, as it was night, and the occurrence was for a few moments unobserved by the attendants. Pressure on the femoral, at the pubic arch, happily sufficed to check any renewal of the bleeding; and for a few days he was most closely watched. However, under the liberal use of stimulants and abundant nourishment, he gradually revived in strength, whilst the wound closed in with marked rapidity. Some suppuration occurred about the situation of the patella, and required relief by an incision; but otherwise the progress of the case, though slow, was satisfactory. The limb was perfectly straight, and the union between the sawn extremities of a fibrous character.

After getting about the ward for some time on crutches, the joint being supported by a splint, he left for fresh air in the country, on September 29th, four months and a half after the operation.

CASE vII. S. E., aged 13, a delicate strumous-looking child, of marked intelligence, was admitted under my care on July 27 th, 1859, with long standing scrofulous disease of the right knee.

History. Two years and a half since, a fall for the first time injured the joint, leading to pain and swelling, and inability to bear weight in standing or walking. Twelve months since, he came under my care in the hospital. At this time the limb was flexed, and of no use. It was straightened under chloroform, and, by the aid of rest and careful bandaging, became so free from pain that he left able to bear his weight, and to get about without the aid of a crutch. However, twelve months passed by, and the knee becoming painful, he was again admitted.

Present State. The joint is very large, globular in shape, acutely painful to the touch, or on the least attempt at movement. It is semiflexed, and the limb above and below is greatly wasted. The child has a hectic appearance, and is much reduced in strength from long continued pain.

August 17th. Under chloroform, I excised the joint in the ordinary way. The operation was quickly accomplished, and there was no hæmorrhage of the least moment. The amount of bone removed altogether from the femur and tibia amounted to about three-quarters of an inch, the epiphysal junction in either bone being untouched. The patella was removed.

Appearances of the Sawn Extremities. The cartilages covering the condyles presented, in places, various stages of destruction, in some being only thinned, and in others entirely removed. The semilunar cartilages of the tibia were entirely gone, and in parts also the cartilages immediately covering the bone. The crucial ligaments were not greatly affected, their external surfaces being only somewhat softened by contiguous mischief. The bones, where sawn through, presented a perfectly healthy section, though the articular extremities were much enlarged from the effects of former disease. In many places, the bone, from which the cartilaginous covering had been entirely removed, was roughened; but in no instance did it appear to be softened. There was no appearance of synovial membrane left. Suppuration was commencing within the joint, but there was none in the textures around.

For two days the boy suffered a little constitutional disturbance. After this subsided, his progress was uninterrupted by any drawback. The wound suppurated and contracted, and the joint presented a solidifying appearance.

September 28 th - six weeks after the operation. The wound is almost bealed. The joint is becoming firm. 'The boy's health is excellent.

At the end of eight weeks, he was permitted to get up, and to move about with the aid of a single crutch and a small leather case. The wound was completely closed, and the boy possessed the most perfect power of raising the entire extremity, the bones having become firmly anchylosed.

\section{[To be continued.]}

\section{DEODORISATION IN CARCINOMA UTERI.}

By Thomas Skinner, M.D., Liverpool.

[Communicated to the Liverpool Medic ll Society, November 3rd, 1350.]

IN perusing the number of the Jounnal for October 29th, I notice a report of a discussion at the Medical Society of London, on the "Deodorisation of Ulcers." I also notice that one of the members present, Dr. Mackenzie, has to a certain extent forestalled me by communicating to the Society the result of his observations in the use of tar water in the treatment of foul ulcers. I am fully aware that priority belongs to neither of us, as Bishop Berkeley believed in it as a panacea for most diseases (Berkeley's Works by Rev. G. N. Wright, vol. ii, p. 308 , et seq.) It has also been highly spoken of by Romberg, Wetherfield, Alibert, Emery, Todd, Bateman, and many other authorities, for internal and external use in cutaneous and pulmonary affections, as well as in the treatment of foul and indolent ulcers. In the treatment of the last mentioned lesions, it has been extolled chiefly, if not entirely, for its stimulant properties, until Dr. Mackenzie's late allusion to it.

With these few preparatory remarks, I beg to draw the attention of my fellow associates to the claims of certain substances as deodorants, particularly tar water, in carcinoma uteri. In the month of June last, a most intelligent and old established practitioner in this town met me in consultation about a lady who is the subject of far advanced scirrhous cancer of the uterus. The cancerous deposition had then extended to the bladder and rectum, rendering the uterus perfectly immobile. The ulcerative process was so far ad. vanced, that the index and middle fingers could be introduced into the cavity, and could be moved freely about. The ulceration also easily admitted Simpson's vaginal speculum, No. 4, an inch and a half in diameter. (This instrument was used twice, simply to apply a solution of the perchloride of iron to abate a termporary hæmorrhage.) The offensiveness of a cancerous discharge from so extensive a surface, and pent up in such a locality, may be more easily imagined than de. scribed. So tenacious and so indestructible were the odorous particles, that soap and chloride of lime failed to deodorise my fingers for hours, and my washhand-stand retained the effluvia for more than forty-eight hours, until I made a free use of McDougall's disinfecting powder, so deservedly praised by Professor Simpson (Medical Times and Gazette, Jan. 22, 1859, p. 80.) The smell was so disgusting to the patient, that she told me she thought " her inside must be like that of a putrid corpse." In such a case, an effectual, speedy, and safe deodorant was a desideratum, if it was not a sine quâ non.

In order to save others the trouble of repeating my ex-

$$
979
$$

